\title{
Mathematica Bohemica
}

Ivan Chajda; Radomír Halaš; Jan Kühr; Alena Vanžurová

Normalization of $M V$-algebras

Mathematica Bohemica, Vol. 130 (2005), No. 3, 283-300

Persistent URL: http://dml.cz/dmlcz/134099

\section{Terms of use:}

(C) Institute of Mathematics AS CR, 2005

Institute of Mathematics of the Czech Academy of Sciences provides access to digitized documents strictly for personal use. Each copy of any part of this document must contain these Terms of use.

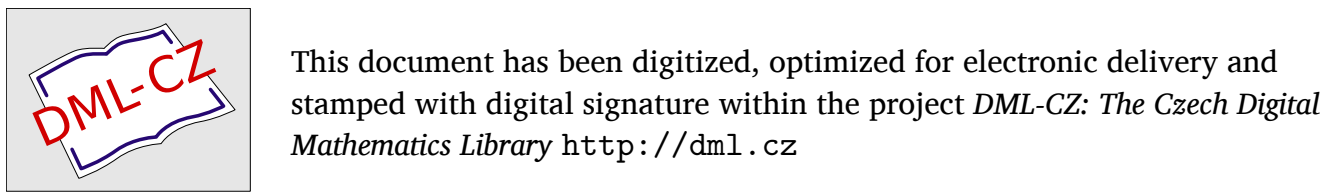




\title{
NORMALIZATION OF $M V$-ALGEBRAS
}

\author{
I. Chajda, R. Halaš, J. Kühr, A. Vanžurová, Olomouc
}

(Received November 19, 2004)

Abstract. We consider algebras determined by all normal identities of $M V$-algebras, i.e. algebras of many-valued logics. For such algebras, we present a representation based on a normalization of a sectionally involutioned lattice, i.e. a $q$-lattice, and another one based on a normalization of a lattice-ordered group.

Keywords: $M V$-algebra, abelian lattice-ordered group, $q$-lattice, normalization of a variety

MSC 2000: 06D35, 06D05, 06F20, 08B20

\section{Preliminaries, normalization, $q$-Lattices}

1.1. Normal identities, normally presentable variety. Let $\tau$ be a similarity type and $X=\left\{x_{1}, x_{2}, \ldots\right\}$ a set of variables. Denote by $T_{\tau}$ the set of all terms of type $\tau$. Let $p, q$ be $n$-ary terms of the given type $\tau$. If either none of them is a variable or both $p, q$ are the same variable, we say that the identity $p\left(x_{1}, \ldots, x_{n}\right)=q\left(x_{1}, \ldots, x_{n}\right)$ is normal.

Let $\mathcal{V}$ be a variety of type $\tau$. Let $\operatorname{Id}(\mathcal{V})$ and $\operatorname{Id}_{N}(\mathcal{V})$ denote the sets of all identities and of all normal identities, respectively, valid in $\mathcal{V}$. The variety $\mathcal{V}$ is called normally presentable if the equality $\operatorname{Id}(\mathcal{V})=\operatorname{Id}_{N}(\mathcal{V})$ holds, cf. [7], [8], [9].

If $\operatorname{Id}(\mathcal{V}) \neq \operatorname{Id}_{N}(\mathcal{V})$ then $\mathcal{V}$ is called here non-normally presentable. If this is the case then there is a unary term $v$ such that the identity $v(x)=x$ belongs to $\operatorname{Id}(\mathcal{V}) \backslash \operatorname{Id}_{N}(\mathcal{V})$, and $\mathcal{V}=\operatorname{Mod}\left(\operatorname{Id}_{N}(\mathcal{V}) \cup\{v(x)=x\}\right)$. As usual, for any set $\Sigma$ of identities of type $\tau, \operatorname{Mod}(\Sigma)$ stands for the class of all algebras of type $\tau$ that satisfy all identities from $\Sigma$.

Supported by grant MSM 6198959214 of the Scientific Council of Czech Government. 
Lemma 1.1. If a non-normally presentable variety $\mathcal{V}$ is given by a system $\Sigma$ of identities, $\mathcal{V}=\operatorname{Mod}(\Sigma)$, and $v(x)=x$ belongs to $\Sigma$, then there exists a system of normal identities valid in $\mathcal{V}, \Sigma_{N} \subset \operatorname{Id}_{N}(\mathcal{V})$, such that $\Sigma_{N} \cup\{v(x)=x\}$ is equivalent to $\Sigma, \mathcal{V}=\operatorname{Mod}\left(\Sigma_{N} \cup\{v(x)=x\}\right)$.

Pro of. Under our assumptions, if a non-normal identity $t\left(x_{1}, \ldots, x_{n}\right)=x_{i}$, $v \neq t \in T_{\tau}$, is satisfied in $\mathcal{V}$ then it can be replaced by the normal identity $t\left(v\left(x_{1}\right), \ldots, v\left(x_{n}\right)\right)=v\left(x_{i}\right)$ which, together with $v(x)=x$, gives back the original one ([11], Proof of Prop. 1, p. 704). Then $\Sigma_{N}$ consisting of all normal identities from $\Sigma$ and of those identities $t\left(v\left(x_{1}\right), \ldots, v\left(x_{n}\right)\right)=v\left(x_{i}\right)$ that replace the non-normal identities $t\left(x_{1}, \ldots, x_{n}\right)=x_{i}$ from $\Sigma$, different from $v(x)=x$, has the required property.

Consequently, $w(x)=x$ is satisfied in $\mathcal{V}$ for another unary term $w$ iff the identity $v(x)=w(x)$ belongs to $\operatorname{Id}_{N}(\mathcal{V})$. So $v$ is determined uniquely up to a normal identity valid in $\mathcal{V}$, and will be called the assigned term of $\mathcal{V},[7]$.

1.2. Normalization. A normalization of $\mathcal{V}$ (called a nilpotent shift of a variety in [7], [9], [11]) is a variety $N(\mathcal{V})$ introduced by $N(\mathcal{V})=\operatorname{Mod}\left(\operatorname{Id}_{N}(\mathcal{V})\right)$. That is, $N(\mathcal{V})$ consists of all $\tau$-algebras which satisfy all normal identities of $\mathcal{V}$. In general $\mathcal{V}$ is a subvariety of $N(\mathcal{V})$, and $\mathcal{V}=N(\mathcal{V})$ holds if and only if the variety is normally presentable.

Corollary 1.2. Let $\mathcal{V}$ be a non-normally presentable variety with an assigned term $v$. Let $\mathcal{N}=\operatorname{Mod}\left(\Xi_{N}\right)$ be a normally presentable variety with the system of defining identities $\Xi_{N} \subset \operatorname{Id}_{N}(\mathcal{V})$. Then $\mathcal{N}=N(\mathcal{V})$ iff all defining identities of $\mathcal{V}$ can be proved from the system $\Xi_{N} \cup\{v(x)=x\}$.

Given a normally presentable variety $\mathcal{N}$ and a non-normal identity $v(x)=x$ then $\mathcal{V}=\operatorname{Mod}(\operatorname{Id}(\mathcal{N}) \cup\{v(x)=x\})$ is the unique variety for which $N(\mathcal{V})=\mathcal{N}$.

Proposition 1.3 ([11], Theorem 2, p. 705). If $\mathcal{V}=\operatorname{Mod}\left(\Sigma_{N} \cup\{v(x)=x\}\right)$ is a variety of type $\tau$ with the set of operation symbols $F$ where $\Sigma_{N} \subset \operatorname{Id}_{N}(\mathcal{V})$ then the normalization is characterized by identities as follows: $N(\mathcal{V})=\operatorname{Mod}\left(\Sigma_{N} \cup \Sigma_{v}\right)$ where the set of additional identities is

$$
\begin{aligned}
\Sigma_{v}=\{ & f\left(x_{1}, \ldots, x_{n}\right)=v\left(f\left(x_{1}, \ldots, x_{n}\right)\right), \\
& \left.f\left(x_{1}, \ldots, x_{j}, \ldots, x_{n}\right)=f\left(x_{1}, \ldots, v\left(x_{j}\right), \ldots, x_{n}\right) ; f \in F, j=1, \ldots, n\right\} .
\end{aligned}
$$

For the proof, see [11], Theorem 1, p. 704 and Lemma, p. 705. 
1.3. Skeleton. Given a non-normally presentable variety $\mathcal{V}$ (of type $\tau$ ) with an assigned term $v$, let $A \in N(\mathcal{V})$. Let us introduce a skeleton of $A$ as a set Sk $A=\{a \in$ $\left.A ; v^{A}(a)=a\right\}$, and call its elements skeletal. Skeletal elements are exactly the results of term operations, i.e. Sk $A=\left\{t^{A}\left(a_{1}, \ldots, a_{n}\right) ; a_{i} \in A, t \in T_{\tau}\right\}$. The algebra $A$ is decomposed into classes $C_{a}=\{d \in A ; v(d)=v(a)\}, a \in$ Sk $A$, called cells of $A$ in [7]. The decomposition is formed exactly by the congruence classes of the congruence relation $\Phi=\left\{\langle a, b\rangle ; t^{A}\left(a, a_{2}, \ldots, a_{n}\right)=t^{A}\left(b, a_{2}, \ldots, a_{n}\right), t \in T_{\tau}, a_{2}, \ldots, a_{n} \in A\right\}$. Moreover, the map $[a]_{\Phi} \mapsto v^{A}(a)$ is an isomorphism $A / \Phi \rightarrow \operatorname{Sk} A$.

Lemma 1.4 ([7], pp.37-38). If $A \in N(\mathcal{V})$ then $\mathrm{Sk} A$ is the maximal subalgebra of $A$ belonging to $\mathcal{V}$.

A construction of a nilpotent shift based on choice algebras is described in [7], [8].

1.4. $q$-lattices as normalization of lattices. A quasiorder on a set $A$ is a reflexive and transitive binary relation $\preceq$ on $A$, and $(A, \preceq)$ is called a quasiordered set.

As is well known, lattices have two faces, can be viewed as algebras and simultaneously as ordered sets. An analogous situation occurs also for algebras resulting from the normalization of lattices, the so-called $q$-lattices. A $q$-lattice can be introduced by identities, but can be characterized as well as a lattice-quasiordered set (with suprema and infima for cells) endowed with a choice function, [6], pp. 7-8.

For our purpose, a variety $\underline{L}$ of lattices can be defined (alternatively) as a variety of type $(2,2)$ and signature $(\vee, \wedge)$ given by the following system of identities (note that only $(\mathrm{I}) \vee$ is not normal):

$$
\begin{aligned}
& \text { commutativity: } \\
& (\mathrm{C})_{\vee}: x \vee y=y \vee x, \quad(\mathrm{C})_{\wedge}: x \wedge y=y \wedge x, \\
& \text { associativity: } \\
& (\mathrm{AS})_{\vee}:(x \vee y) \vee z=x \vee(y \vee z), \quad(\mathrm{AS})_{\wedge}:(x \wedge y) \wedge z=x \wedge(y \wedge z), \\
& \text { weak absorption: } \\
& (\mathrm{WAB})_{\vee}: x \vee(x \wedge y)=x \vee x \text {, } \\
& \text { idempotency: } \\
& (\mathrm{I})_{\vee}: x \vee x=x \text {, } \\
& (\mathrm{WAB})_{\wedge}: x \wedge(x \vee y)=x \wedge x \text {, } \\
& \text { equalization: } \\
& \text { (EQ): } x \wedge x=x \vee x \text {. }
\end{aligned}
$$

The variety $\underline{L}$ is not normally presentable, we can choose e.g. $v(x)=x \vee x$ as an assigned term (or equivalently, $x \wedge x$, [8], p.328), and construct the normalization. Since there is a single non-normal identity among the defining ones we 
can apply the general theory to obtain $N(\underline{L})=\operatorname{Mod}\left(\Sigma_{N} \cup \Sigma_{v}\right)$ where $\Sigma_{N}=$ $\left\{(\mathrm{C})_{\wedge},(\mathrm{C})_{\vee},(\mathrm{AS})_{\wedge},(\mathrm{AS})_{\vee},(\mathrm{EQ}),(\mathrm{WAB})_{\wedge},(\mathrm{WAB})_{\vee}\right\}$ and $\Sigma_{v}$ consists of the identities

$$
\begin{aligned}
& \Sigma_{v}: x \vee y=(x \vee x) \vee y, \quad x \vee y=x \vee(y \vee y), \quad(x \vee y) \vee(x \vee y)=x \vee y, \\
& x \wedge y=(x \vee x) \wedge y, \quad x \wedge y=x \wedge(y \vee y), \quad(x \wedge y) \vee(x \wedge y)=x \wedge y
\end{aligned}
$$

In [6], the variety of $q$-lattices was introduced as

$$
\operatorname{Mod}\left(\left\{(\mathrm{C})_{\vee},(\mathrm{C})_{\wedge},(\mathrm{AS})_{\vee},(\mathrm{AS})_{\wedge},(\mathrm{WAB})_{\vee},(\mathrm{WAB})_{\wedge},(\mathrm{EQ}),(\mathrm{WI})_{\vee},(\mathrm{WI})_{\wedge}\right\}\right)
$$

where

$$
(\mathrm{WI})_{\vee}: x \vee y=x \vee(y \vee y), \quad(\mathrm{WI})_{\wedge}: x \wedge y=x \wedge(y \wedge y) \quad \text { (weak idempotency) }
$$

(see also [7], [8] etc.). It can be easily seen that $N(\underline{L})$ is exactly the variety of the $q$ lattices. In fact, $(\mathrm{WI}) \wedge$ follows immediately from the identities of $N(\underline{L})$ (and $(\mathrm{WI})_{\vee}$ is among the defining ones). Vice versa, if $(\mathrm{WI})_{\vee}$ holds then by $(\mathrm{C})_{\vee}$ and $(\mathrm{AS})_{\vee}$, $(x \vee x) \vee y=y \vee(x \vee x)=y \vee x=x \vee y$ and $(x \vee y) \vee(x \vee y)=x \vee(x \vee(y \vee y))=$ $x \vee(x \vee y)=x \vee y$. If $(\mathrm{WI}) \wedge$ is satisfied then also $x \wedge y=(x \wedge x) \wedge y$ holds, $(x \vee x) \wedge y=(x \wedge x) \wedge y=x \wedge y$ follows by (EQ), and by (EQ) and duality, $x \wedge(y \vee y)=x \wedge(y \wedge y)=x \wedge y,(x \wedge y) \vee(x \wedge y)=(x \wedge y) \wedge(x \wedge y)=x \wedge y$.

An algebra $(A, \vee)$ satisfying the identities $(\mathrm{C})_{\vee},(\mathrm{AS})_{\vee}$ and $(\mathrm{WI})_{\vee}$ is called a join$q$-semilattice. A $q$-lattice is called distributive if it satisfies the distributive identity or its dual (which are both normal).

\section{Normalization of $M V$-Algebras}

An $M V$-algebra is an algebra $\mathcal{A}=(A, \oplus, \neg, 0)$ of type $(2,1,0)$ satisfying the identities

(MV1) $x \oplus(y \oplus z)=(x \oplus y) \oplus z$,

(MV2) $x \oplus y=y \oplus x$,

(MV3) $x \oplus 0=x$,

(MV4) $\neg \neg x=x$,

(MV5) $x \oplus \neg 0=\neg 0$,

(MV6) $\neg(\neg x \oplus y) \oplus y=\neg(\neg y \oplus x) \oplus x$.

Clearly, also the (normal) identities $\neg \neg x=x \oplus 0$ and $\neg \neg \neg x=\neg x$ hold.

$M V$-algebras were introduced as an algebraic tool for many valued logics, [2]. They were studied as an algebraic counterpart of the Lukasziewicz infinite valued propositional logic, [3], [12]. Later on, a close connection to other structures 
was discovered, namely to lattice-ordered abelian groups, [4], bounded commutative $B C K$-algebras, [13], and bounded $D R l$-semigroups, [14] etc. The $M V$-algebras form a variety $\underline{M V}=\operatorname{Mod}(\{(\mathrm{MV} 1)-(\mathrm{MV} 6)\})$ that is not normally presentable, with $v(x)=x \oplus 0$ as an assigned term (or equivalently, $v^{\prime}(x)=\neg \neg x$ ). According to Proposition 1.3, the normalization $N(\underline{M V})$ has a basis consisting of the following normal identities: (MV1), (MV2), (MV5), (MV6), $\neg \neg x=x \oplus 0, x \oplus y=(x \oplus 0) \oplus y$, $x \oplus y=x \oplus(y \oplus 0),(x \oplus y) \oplus 0=x \oplus y, \neg(x \oplus 0)=\neg x, \neg x \oplus 0=\neg x, 0 \oplus 0=0$.

Denote

(N1) $x \oplus(y \oplus z)=(x \oplus y) \oplus z$,

(N2) $x \oplus y=y \oplus x$,

(N3) $0 \oplus 0=0$,

(N4) $\neg \neg x=x \oplus 0$,

(N5) $x \oplus \neg 0=\neg 0$,

(N6) $\neg(\neg x \oplus y) \oplus y=\neg(\neg y \oplus x) \oplus x$,

(N7) $\neg(x \oplus 0)=\neg x$,

(N8) $\neg x \oplus 0=\neg x$,

(N9) $(x \oplus y) \oplus 0=x \oplus y$.

Then $N(\underline{M V})=\operatorname{Mod}\left(\operatorname{Id}_{N}(\underline{M V})\right)=\operatorname{Mod}(\{(\mathrm{N} 1)-(\mathrm{N} 9)\})$. Further, denote

$\left(\mathrm{N} 4^{\prime}\right) \neg \neg 0=0 \oplus 0$,

(N10) $\neg \neg \neg x=\neg x$.

Lemma 2.1. The following implications hold:

(i) (N4) (or $\left(\mathrm{N} 4^{\prime}\right)$ ) and (N3) imply $\neg \neg 0=0$,

(ii) (N7) and (N8) imply $\neg x \oplus 0=\neg(x \oplus 0)$,

(iii) (N2), (N3), (N4'), (N5)-(N9) imply (N4),

(iv) (N4) and (N7) imply (N10),

(v) (N10) and (N4) imply (N7), (N8).

Proof. The first two cases are obvious. Let us verify (iii). (N8) used for $\neg x$ yields $\neg \neg x=\neg \neg x \oplus 0$. Using (ii), (N9), (N6), (N2), (N5) and (i) we obtain $\neg \neg x \oplus 0=\neg(\neg x \oplus 0) \oplus 0=\neg(\neg 0 \oplus x) \oplus x=\neg(\neg 0) \oplus x=0 \oplus x=x \oplus 0$, proving (iii). Now (N4) and (N7) yield $\neg \neg \neg x=\neg(x \oplus 0)=\neg x$, and (iv) holds. Suppose (N10) and (N4) are satisfied. Then $\neg x=\neg(\neg \neg x)=\neg(x \oplus 0)$, similarly $\neg x=\neg \neg(\neg x)=\neg x \oplus 0$, and (v) holds.

So $N(\underline{M V})=\operatorname{Mod}\left(\left\{(\mathrm{N} 1)-(\mathrm{N} 3),\left(\mathrm{N} 4^{\prime}\right),(\mathrm{N} 5),(\mathrm{N} 6),(\mathrm{N} 9),(\mathrm{N} 10)\right\}\right)$. The skeleton of $(M, \oplus, \neg, 0)$ from $(\underline{M V})$ is $\operatorname{Sk} M=\{a \in M ; a=a \oplus 0\}$, and (Sk $M, \oplus, \neg, 0)$ is an $M V$-algebra.

It is well-known (see [5]) that $M V$-algebras with respect to a natural order defined by $x \leqslant y$ iff $\neg x \oplus y=\neg 0$ form a bounded distributive lattice where $x \vee y=\neg(\neg x \oplus$ 
$y) \oplus y$ and $x \wedge y=\neg(\neg x \vee \neg y)$. A similar statement can be formulated for their normalizations:

Proposition 2.2. Let $\mathcal{A}=(A, \oplus, \neg, 0) \in N(\underline{M V})$. Define $x \preceq y$ iff $\neg x \oplus y=\neg 0$. Then $(A, \preceq)$ is a bounded distributive $q$-lattice with 0 as the least element and $\neg 0$ as the greatest one, in which $x \vee y=\neg(\neg x \oplus y) \oplus y$ and $x \wedge y=\neg(\neg x \vee \neg y)$.

Proof. The proof follows from the fact that the operations $\vee$ and $\wedge$ satisfy all normal identities of a lattice, hence $(A, \preceq)$ is a $q$-lattice. Moreover, a $q$-lattice is distributive iff its skeleton is distributive.

\section{Normalization of $l$-GROUPS}

$M V$-algebras can be represented as intervals in abelian lattice-ordered groups, see [12]. We are going to prove an analogous statement for algebras from the normalization $N(\underline{M V})$ of the variety $\underline{M V}$; up to isomorphism, any algebra $M \in N(\underline{M V})$ can be realized on a suitable section (= interval) of some algebra from the normalization of the variety of (abelian) $l$-groups.

An abelian lattice-ordered group, shortly an l-group, is an algebra $\mathcal{G}=(G,+,-$, $0, \vee, \wedge)$ of type $(2,1,0,2,2)$ such that $(G,+,-, 0)$ is an abelian group, $(G, \vee, \wedge)$ is a lattice (with induced order $\leqslant$ ) and + distributes with each of the operations $\vee$ and $\wedge$. That is, $\mathcal{G}$ is an (abelian) $l$-group if and only if it fulfils the identities

(A1) $x+y=y+x$,

(A2) $(x+y)+z=x+(y+z)$,

(A3) $x+0=x$,

(A4) $x+(-x)=0$,

(A5) $x \vee y=y \vee x, x \wedge y=y \wedge x$,

(A6) $(x \vee y) \vee z=x \vee(y \vee z), \quad(x \wedge y) \wedge z=x \wedge(y \wedge z)$,

(A7) $x \vee(x \wedge y)=x, x \wedge(x \vee y)=x$,

(A8) $x \vee x=x, \quad x \wedge x=x$,

(A9) $(x \vee y)+z=(x+z) \vee(y+z), \quad(x \wedge y)+z=(x+z) \wedge(y+z)$.

One readily sees that $(\mathrm{A} 7)$ and $(\mathrm{A} 8)$ can be equivalently replaced by normal identities

$\left(\mathrm{A} 7^{\prime}\right) x \vee(x \wedge y)=x \vee x, x \wedge(x \vee y)=x \wedge x$,

$\left(\mathrm{A} 8^{\prime}\right) x \vee x=x+0, \quad x \wedge x=x+0$.

Now the only non-normal identity is (A3). The variety of $l$-groups

$$
\underline{L G}=\operatorname{Mod}\left(\left\{(\mathrm{A} 1)-(\mathrm{A} 6),\left(\mathrm{A} 7^{\prime}\right),\left(\mathrm{A} 8^{\prime}\right),(\mathrm{A} 9)\right\}\right)
$$


is not normally presentable, we can take e.g. $v(x)=x+0$ as an assigned term of $\underline{L G}$ (or equivalently, $w(x)=x \wedge x$, or $w^{\prime}(x)=x \vee x$ ), and construct the normalization. Let $\Sigma_{N}$ be the set consisting of (A1), (A2), (A4)-(A6), (A7'), (A8'), (A9). Due to Proposition 1.3, $N(\underline{L G})=\operatorname{Mod}\left(\Sigma_{N} \cup \Sigma_{v}\right)$ where $\Sigma_{v}$ consists of the identities

(A10) $x+y=x+y+0$,

(A11) $-x=-(x+0)$,

(A12) $-x=-x+0$,

(A13) $x \vee y=(x+0) \vee y$,

(A14) $x \wedge y=(x+0) \wedge y$,

(A15) $x \vee y=(x \vee y)+0$,

(A16) $x \wedge y=(x \wedge y)+0$,

(A17) $0+0=0$

(and of the identities $x \vee y=x \vee(y+0), x \wedge y=x \wedge(y+0)$ that can be omitted since they easily follow from (A13), (A14) by interchanging $x, y$ and using commutativity). (A15) follows from (A9) and (A13), $(x \vee y)+0=(x+0) \vee(y+0)=x \vee(y+0)=x \vee y$. Similarly, (A16) can be proved from (A9) and (A14). Moreover, by (A4) and (A10) we get $0+0=x+(-x)+0=x+(-x)=0$. We have obtained

Proposition 3.1. The normalization $N(\underline{L G})=\operatorname{Mod}\left(\operatorname{Id}_{N}(\underline{L G})\right)$ of $\underline{L G}$ is

$$
N(\underline{L G})=\operatorname{Mod}\left(\left\{(\mathrm{A} 1),(\mathrm{A} 2),(\mathrm{A} 4)-(\mathrm{A} 6),\left(\mathrm{A} 7^{\prime}\right),\left(\mathrm{A} 8^{\prime}\right),(\mathrm{A} 9)-(\mathrm{A} 14)\right\}\right) .
$$

To emphasize the expected fact that $(G, \vee, \wedge)$ is a $q$-lattice whenever $\mathcal{G}=$ $(G,+,-, 0, \vee, \wedge)$ belongs to $N(\underline{L G})$ we can use

$$
\left(\mathrm{A}^{\prime} 3^{\prime}\right) x \vee y=x \vee x \vee y
$$

and

$\left(\mathrm{A} 14^{\prime}\right) x \wedge y=x \wedge x \wedge y$

instead of (A13) and (A14), respectively.

Remark 3.2. It is well known that the lattice of an l-group is distributive (e.g. [1], p.3). Since distributivity is a normal identity, the $q$-lattice corresponding to an algebra from $N(\underline{L G})$ is distributive as well.

Given an algebra $\mathcal{G} \in N(\underline{L G})$, the skeleton $\operatorname{Sk} \mathcal{G}=\{a \in G ; a=a+0\}=\{a \in$ $G ; a=a \wedge a\}$ is the carrier set of an $l$-group $(\operatorname{Sk} \mathcal{G},+,-, 0, \vee, \wedge)$.

Since $(G, \vee, \wedge)$ is a $q$-lattice, the binary relation $\preceq$ defined by $x \preceq y$ iff $x \wedge x=x \wedge y$ (or equivalently, $x \preceq y$ iff $x \vee y=y \vee y$ ) is a quasiorder on $G$.

Let us verify that (right) translations $R_{z}: x \mapsto x+z$ are isotone with respect to this quasiorder. 
Lemma 3.3. If $x \preceq y$ then $x+z \preceq y+z$.

Proof. Let $x \preceq y$, i.e. $x \wedge x=x \wedge y$. Then

$$
(x+z) \wedge(x+z)=(x \wedge x)+z=(x \wedge y)+z=(x+z) \wedge(y+z)
$$

so that $x+z \preceq y+z$.

Similarly, the operations $\wedge$ and $\vee$ are isotone.

Lemma 3.4. If $x \preceq y$ then $x \wedge z \preceq y \wedge z$ and $x \vee z \preceq y \vee z$.

Pro of. $\quad$ Let $x \preceq y$, i.e. $x \wedge x=x \wedge y$. Then by $(\mathrm{AS})_{\wedge}$ and $(\mathrm{C})_{\wedge},(x \wedge z) \wedge(x \wedge z)=$ $(x \wedge x) \wedge(z \wedge z)=(x \wedge y) \wedge(z \wedge z)=(x \wedge z) \wedge(y \wedge z)$, i.e. $x \wedge z \preceq y \wedge z$. By distributivity, $(x \vee z) \wedge(x \vee z)=(x \wedge x) \vee z=(x \wedge y) \vee z=(x \vee z) \wedge(y \vee z)$, so $x \vee z \preceq y \vee z$.

Let $\mathcal{G} \in N(\underline{L G})$. Given $u \in G, u \succeq 0$ denote $[0, u]=\{x \in G ; 0 \preceq x \preceq u\}$. On $[0, u]$, a structure of an algebra from $N(\underline{M V})$ arises as follows.

Theorem 3.5. Let $\mathcal{G} \in N(\underline{L G})$ and let $u \in \operatorname{Sk} \mathcal{G}, 0 \preceq u$. Define

$$
a \oplus b:=(a+b) \wedge u, \quad \neg a:=u+(-a)
$$

for $a, b \in[0, u]$. Then the algebra $\Gamma(\mathcal{G}, u):=([0, u], \oplus, \neg, 0)$ belongs to $N(\underline{M V})$.

From now on, let us write $x-y$ for $x+(-y)$.

Pro of. Let $a, b \in[0, u]$. By Lemma 3.3 we have $0=0+0 \preceq a+b$, and hence $0=0 \wedge 0=0 \wedge u \preceq(a+b) \wedge u=a \oplus b$, so $a \oplus b \preceq u$ proving $a \oplus b \in[0, u]$.

Further, $(u-a) \wedge 0=(u-a) \wedge(a-a)=(u \wedge a)-a=(a \wedge a)-a=(a-a) \wedge(a-a)=$ $0 \wedge 0=0$, i.e. $0 \preceq u-a$, and similarly, $(u-a) \vee u=(u-a) \vee(u+0)=u+(-a \vee 0)=$ $u-(a \wedge 0)=u-(0 \wedge 0)=u-0=u$, i.e. $u-a \preceq u$ proving $\neg a \in[0, u]$. We have used $-0=-0+0=(-0+0)+0=0+0=0$ and the normal identity $-(x \vee y)=(-x) \wedge(-y)$. Now let us verify $(\mathrm{N} 1)-(\mathrm{N} 9)$.

$(\mathrm{N} 1):(x \oplus y) \oplus z=(((x+y) \wedge u)+z) \wedge u=(x+y+z) \wedge(u+z) \wedge u=(x+y+z) \wedge u$ since $u=u+0 \preceq u+z$ by Lemma 3.3. Analogously, we evaluate $x \oplus(y \oplus z)=$ $(x+((y+z) \wedge u)) \wedge u=(x+y+z) \wedge(x+u) \wedge u=(x+y+z) \wedge u$.

(N2) follows by commutativity of + .

(N3): $0 \oplus 0=(0+0) \wedge u=0 \wedge u=0$.

(N4): $\neg \neg x=u-(u-x)=u-u+x=0+x=x+0$; we have used the normal identity $x-(y-z)=x-y+z$.

(N5): $x \oplus \neg 0=(x+u-0) \wedge u=(x+u) \wedge u=u$ and $\neg 0=u-0=u+0=u$. 
(N6):

$$
\begin{aligned}
\neg(\neg x \oplus y) \oplus y & =(u-((u-x+y) \wedge u)+y) \wedge u \\
& =((u-u+x-y+y) \vee(u-u+y)) \wedge u \\
& =((0+x+0) \vee(0+y)) \wedge u \\
& =((x+0) \vee(y+0)) \wedge u \\
& =(x \vee y) \wedge u=x \vee y
\end{aligned}
$$

and analogously, by replacing $x$ and $y$ and using commutativity, $\neg(\neg y \oplus x) \oplus x=$ $y \vee x=x \vee y$.

(N7): $\neg(x \oplus 0)=u-((x+0) \wedge u)=(u-(x+0)) \vee(u-u)=(u-x) \vee 0=u-x=\neg x$.

(N8): $\neg x \oplus 0=(u-x+0) \wedge u=(u-x) \wedge u=u-x=\neg x$.

(N9) is evident.

In the theorem, if $\mathcal{G}$ is an $l$-group then $\Gamma(\mathcal{G}, u)$ is an $M V$-algebra. By D. Mundici's famous result on $M V$-algebras and $l$-groups, [12], every $M V$-algebra is isomorphic to $\Gamma(\mathcal{G}, u)$ for some (abelian) $l$-group $\mathcal{G}$ with a strong order unit ${ }^{1} u$, [5].

We are going to show that any algebra belonging to the normalization $N(\underline{M V})$ of the variety of $M V$-algebras can be obtained in the way described in Theorem 3.5 as $\Gamma\left(\mathcal{G}^{*}, u\right)$ for a suitable $\mathcal{G}^{*}$ from the normalization $N(\underline{L G})$ of abelian $l$-groups and a suitable $u \in \mathrm{Sk}_{\mathcal{G}^{*}}$ with $0 \preceq u$.

So let $\mathcal{A}=(A, \oplus, \neg, 0)$ be an algebra from $N(\underline{M V})$. Then $(\operatorname{Sk} \mathcal{A}, \oplus, \neg, 0)$ is an $M V$-algebra and we may assume that the skeleton $\operatorname{Sk} \mathcal{A}$ equals $\Gamma(\mathcal{G}, u)$ for some $l$-group $\mathcal{G}=\left(G,+_{G},-_{G}, 0, \vee_{G}, \wedge_{G}\right)$ and a strong unit $u \in G$.

Let $G^{*}=G \cup A$ and let us define binary operations $\vee, \wedge,+$, and a unary operation - on $G^{*}$ as follows:

$$
x \vee y:= \begin{cases}x \vee_{G} y & \text { if } x, y \in G, \\ \left(x \vee_{A} x\right) \vee_{G} y & \text { if } x \in G^{*} \backslash G, y \in G, \\ x \vee_{G}\left(y \vee_{A} y\right) & \text { if } x \in G, y \in G^{*} \backslash G, \\ x \vee_{A} y & \text { if } x, y \in G^{*} \backslash G,\end{cases}
$$

$x \wedge y$ is defined dually,

$$
\begin{aligned}
x+y & := \begin{cases}x+{ }_{G} y & \text { if } x, y \in G, \\
\left(x \vee_{A} x\right)+{ }_{G} y & \text { if } x \in G^{*} \backslash G, y \in G, \\
x+{ }_{G}\left(y \vee_{A} y\right) & \text { if } x \in G, y \in G^{*} \backslash G, \\
\left(x \vee_{A} x\right)+{ }_{G}\left(y \vee_{A} y\right) & \text { if } x, y \in G^{*} \backslash G,\end{cases} \\
-x & := \begin{cases}-{ }_{G} x & \text { if } x \in G, \\
-{ }_{G}\left(x \vee_{A} x\right) & \text { if } x \in G^{*} \backslash G .\end{cases}
\end{aligned}
$$

\footnotetext{
${ }^{1}$ An element $u \in G$ is called a strong order unit if $0 \leqslant u$ and for any $x \in G$ there exists $k \in \mathbb{N}$ such that $x \leqslant k \cdot u$.
} 
A tedious but straightforward verification yields that the structure $\mathcal{G}^{*}=\left(G^{*},+\right.$, $-, 0, \vee, \wedge)$ satisfies all the identities (A1), (A2), (A4)-(A6), (A7'), (A8'), (A9)-(A14). In addition, $[0, u]=A, \operatorname{Sk} \mathcal{G}^{*}=G$ and $u \in \operatorname{Sk} \mathcal{G}^{*}$, and in $\Gamma\left(\mathcal{G}^{*}, u\right)=\left(A, \oplus^{*}, \neg^{*}, 0\right)$, where $x \oplus^{*} y=(x+y) \wedge u$ and $\neg^{*} x=u-x$, we have

$$
x \oplus^{*} y=(x+y) \wedge u= \begin{cases}\left(x+{ }_{G} y\right) \wedge_{G} y=x \oplus y & \text { if } x, y \in G, \\ \left(\left(x \vee_{A} x\right)+_{G} y\right) \wedge_{G} u=\left(x \vee_{A} x\right) \oplus y & \\ =(x \oplus y) \vee_{A}(x \oplus y)=x \oplus y & \text { if } x \in G^{*} \backslash G, y \in G, \\ \left(x+{ }_{G}\left(y \vee_{A} y\right)\right) \wedge_{G} u=x \oplus y & \text { if } x \in G, y \in G^{*} \backslash G, \\ \left(\left(x \vee_{A} x\right)+_{G}\left(y \vee_{A} y\right)\right) \wedge_{G} u & \\ =\left(x \vee_{A} x\right) \oplus\left(y \vee_{A} y\right)=x \oplus y & \text { if } x, y \in G^{*} \backslash G\end{cases}
$$

and

$$
\neg^{*} x=u-x= \begin{cases}u-{ }_{G} x=\neg x & \text { if } x \in G, \\ u-{ }_{G}\left(x \vee_{A} x\right)=\neg\left(x \vee_{A} x\right)=\neg x & \text { if } x \in G^{*} \backslash G .\end{cases}
$$

Therefore $(A, \oplus, \neg, 0)$ is isomorphic to $\Gamma\left(\mathcal{G}^{*}, u\right)$. We have proved

Theorem 3.6. For any algebra $\mathcal{A} \in N(\underline{M V})$ there exists an algebra $\mathcal{G} \in N(\underline{L G})$ and an element $u \in \operatorname{Sk} \mathcal{G}, 0 \preceq u$ such that $\mathcal{A}$ is isomorphic to $\Gamma(\mathcal{G}, u)$.

\section{4. $q$-LATTICES WITH SECTIONALLY ANTITONE INVOLUTIONS}

As usual, under an involution on a set $A$ we mean a map $f: A \rightarrow A$ such that $f(f(a))=a$ for all $a \in A$.

Given a quasiordered set $(A, \preceq)$, a map ${ }^{p}: A \rightarrow A$ is called antitone if the implication $x \preceq y \Longrightarrow y^{p} \preceq x^{p}$ holds.

Let $\mathcal{L}=(L, \vee, \wedge, 1)$ be a $q$-lattice with the greatest idempotent $1,1=1 \vee 1$, and let $\preceq$ denote the induced quasiorder on $L$. Note that the skeleton $\operatorname{Sk} \mathcal{L}=\{x \in$ $L ; x \vee x=x\}$ is a lattice. Under an interval in $\mathcal{L}$ we understand here the set $[a, b]=\{x \in L ; a \preceq x \preceq b\}$, and under an interval in the skeleton the intersection $\operatorname{Sk}[a, b]=\operatorname{Sk} L \cap[a, b]$ provided $a, b \in \operatorname{Sk} L$.

Rem ark 4.1. For any $p \in L$, let an antitone involution ${ }^{p}: x \mapsto x^{p}, x \in \operatorname{Sk} L$, be given on the interval $\operatorname{Sk}[p \vee p, 1]$. The mapping ${ }^{p}$ with $p \in L$ can be extended to a mapping of the whole interval (denoted by the same symbol) ${ }^{p}:[p, 1] \rightarrow[p, 1]$, $x \mapsto x^{p}$, in a natural way as follows. For $x \in[p, 1]$ define $x^{p}:=(x \vee x)^{p \vee p}$. Note that in general, ${ }^{p}$ is not an involution on $[p, 1]$ but only on $\operatorname{Sk}[p \vee p, 1]$. Indeed, $x^{p p}=\left((x \vee x)^{p \vee p} \vee(x \vee x)^{p \vee p}\right)^{p \vee p}=\left((x \vee x)^{p \vee p}\right)^{p \vee p}=x \vee x \in$ Sk $L$, i.e. $x^{p p} \neq x$ if $x \notin \mathrm{Sk} L$. But nevertheless, we get $x^{p p p}=(x \vee x)^{p \vee p}=x^{p}$ as a consequence. 
Lemma 4.2. Let $\mathcal{L}=(L, \vee, \wedge, 1)$ be a $q$-lattice, $1=1 \vee 1$. For any $p \in L$, let an antitone involution ${ }^{p}: x \mapsto x^{p}, x \in \operatorname{Sk} L$, be given on the interval $\operatorname{Sk}[p \vee p, 1]$. For $x$, $y \in L$, let us introduce a binary operation $x \circ y:=(x \vee y)^{y \vee y}$. Then the following identities hold:

(1) $x \circ 1=1, x \circ x=1$,

(2) $1 \circ(x \circ y)=x \circ y$,

(3) $\quad(x \circ y) \circ y=(y \circ x) \circ x$ (quasi-commutativity).

Proof. Indeed, $x \circ x=(x \vee x)^{x \vee x}=1, x \circ 1=(x \vee 1)^{1 \vee 1}=1^{1}=1$, $1 \circ(x \circ y)=1 \circ(x \vee y)^{y \vee y}=\left(1 \vee(x \vee y)^{y \vee y}\right)^{(x \vee y)^{y \vee y}}=1^{(x \vee y)^{y \vee y}}=x \circ y$. Further, $(x \circ y) \circ y=\left((x \vee y)^{y \vee y} \vee y\right)^{y \vee y}$. Here $(x \vee y)^{y \vee y} \vee y=(x \vee y)^{y \vee y}$ since $(x \vee y)^{y \vee y} \succeq$ $y \vee y \succeq y$, therefore $\left((x \vee y)^{y \vee y} \vee y\right)^{y \vee y}=x \vee y$, and (2) follows.

Definition 4.3. Under a normal chain in a $q$-lattice we understand a sequence $a_{0}, \ldots, a_{n}, \ldots$ of elements from $L$ such that $a_{0} \succ a_{1} \succ \ldots \succ a_{n} \succ \ldots$

Proposition 4.4. Let $L=\left\{a_{0}=1, a_{1}, \ldots, a_{n}, \ldots\right\}$ be a normal chain. Then the lattice $\operatorname{Sk} L=\left\{a_{0} \vee a_{0}, a_{1} \vee a_{1}, \ldots, a_{n} \vee a_{n}, \ldots\right\}$ is a lattice with sectionally antitone involutions in which for $a_{i} \vee a_{i} \in\left[a_{n} \vee a_{n}, a_{0} \vee a_{0}\right]$, there is a (unique) involution given by $\left(a_{i} \vee a_{i}\right)^{a_{n} \vee a_{n}}=a_{n-i} \vee a_{n-i}$. If we introduce

$$
a_{i} \circ a_{j}=\left(a_{i} \vee a_{j}\right)^{a_{j} \vee a_{j}} \text { for } a_{i}, a_{j} \in L
$$

then the identity

$$
x \circ(y \circ z)=y \circ(x \circ z) \quad(\text { exchange })
$$

holds in $L$.

Proof. Let $a_{i}, a_{j}, a_{k} \in L$. If $a_{j} \preceq a_{k}$ then $a_{j} \circ a_{k}=\left(a_{j} \vee a_{k}\right)^{a_{k} \vee a_{k}}=$ $\left(a_{k} \vee a_{k}\right)^{a_{k} \vee a_{k}}=1 \vee 1$, that is $a_{i} \circ\left(a_{j} \circ a_{k}\right)=\left(a_{i} \vee 1 \vee 1\right)^{1 \vee 1}=(1 \vee 1)^{1 \vee 1}=1 \vee 1$. If $a_{i} \preceq a_{k}$ we obtain the equality again in a similar way. So we can suppose $a_{i} \succeq a_{j} \succeq a_{k}$. Then

$$
\begin{aligned}
a_{i} \circ\left(a_{j} \circ a_{k}\right) & =a_{i} \circ\left(a_{j} \vee a_{k}\right)^{a_{k} \vee a_{k}} \\
& =a_{i} \circ\left(a_{j} \vee a_{j}\right)^{a_{k} \vee a_{k}}=a_{i} \circ\left(a_{k-j} \vee a_{k-j}\right)=\left(a_{i} \vee a_{k-j}\right)^{a_{k-j} \vee a_{k-j}} \\
& =\left\{\begin{array}{l}
1, \quad i \geqslant k-j, \\
\left(a_{i} \vee a_{i}\right)^{a_{k-j} \vee a_{k-j}}=a_{k-j-i} \vee a_{k-j-i}, \quad i<k-j .
\end{array}\right.
\end{aligned}
$$

Taking into account symmetry, we obtain

$$
a_{j} \circ\left(a_{i} \circ a_{k}\right)=a_{j} \circ\left(a_{k-i} \vee a_{k-i}\right)=\left\{\begin{array}{l}
1, \quad j \geqslant k-i, \\
a_{k-i-j} \vee a_{k-i-j}, \quad j<k-i .
\end{array}\right.
$$

Since $i \geqslant k-j$ is equivalent to $j \geqslant k-i$, the equality $a_{i} \circ\left(a_{j} \circ a_{k}\right)=a_{j} \circ\left(a_{i} \circ a_{k}\right)$ holds. 
Lemma 4.5. Let $(A, \circ, 1)$ be an algebra of type $(2,0)$ satisfying the identities $(1)$, (2), (3) and (4). Then

(i) $((x \circ y) \circ y) \circ y=x \circ y$;

(ii) the relation $\preceq$ introduced by

$$
x \preceq y \Longleftrightarrow x \circ y=1
$$

is a quasiorder on $A$ and for all $x \in A$, we have $x \preceq 1$;

(iii) the right translations $R_{z}^{\circ}: x \mapsto x \circ z, z \in A$, are antitone with respect to the quasiorder, while the left translations $L_{z}^{\circ}: x \mapsto x \circ z$ are isotone, that is,

$$
x \preceq y \Longrightarrow y \circ z \preceq x \circ z, \quad z \circ x \preceq z \circ y, \quad x, y, z \in A
$$

Proof. According to (1) and (4), $y \circ(x \circ y)=x \circ(y \circ y)=x \circ 1=1$; further $((x \circ y) \circ y) \circ y=(y \circ(x \circ y)) \circ(x \circ y)$ by (4). Hence by $(2),((x \circ y) \circ y) \circ y=1 \circ(x \circ y)=x \circ y$, and (i) holds.

By $(1)$, $\preceq$ is reflexive. Let us prove transitivity. Let $x \preceq y, y \preceq z$, that is, $x \circ y=y \circ z=1$. Then by (2), (4) and (3), $x \circ z=1 \circ(x \circ z)=x \circ(1 \circ z)=$ $x \circ((y \circ z) \circ z)=x \circ((z \circ y) \circ y)=(z \circ y) \circ(x \circ y)=(z \circ y) \circ 1=1$, so that $x \preceq z$, and (iii) holds. Now if $x \preceq y$ then $x \circ y=1$ and due to (4), (3) and (1) we have $(y \circ z) \circ(x \circ z)=x \circ((y \circ z) \circ z)=x \circ((z \circ y) \circ y)=(z \circ y) \circ(x \circ y)=(z \circ y) \circ 1=1$. Therefore $y \circ z \preceq x \circ z$. Moreover, by (2), (4), (3) we have $z \circ y=1 \circ(z \circ y)=$ $(x \circ y) \circ(z \circ y)=z \circ((x \circ y) \circ y)=z \circ((y \circ x) \circ x)=(y \circ x) \circ(z \circ x)$, consequently (by (4), (1)) $(z \circ x) \circ(z \circ y)=(z \circ x) \circ[(y \circ x) \circ(z \circ x)]=(y \circ x) \circ 1=1$, hence $z \circ x \preceq z \circ y$, which completes the proof of (iii).

The quasiorder $\preceq$ given by $x \preceq y \Longleftrightarrow x \circ y=1$ will be called the induced quasiorder on $(A, \circ, 1)$.

Proposition 4.6. Let $\mathcal{A}=(A, \circ, 1)$ be an algebra satisfying the identities (1)-(4). If $x \vee y:=(x \circ y) \circ y$ then $(A, \preceq)$ is a join- $q$-semilattice with the greatest element 1. For any $p \in A$, the interval $[p \vee p, 1]$ is a distributive $q$-lattice with an antitone involution

$$
a \mapsto a^{p}=a \circ p, \quad a \in[p \vee p, 1], \quad a \in \operatorname{Sk}[p \vee p, 1]
$$

Proof. For $x, y \in A, y \circ((x \circ y) \circ y)=(x \circ y) \circ(y \circ y)=(x \circ y) \circ 1=1$ holds by (4) and (1), similarly $x \circ((x \circ y) \circ y)=(x \circ y) \circ(x \circ y)=1$, hence $(x \circ y) \circ y$ is an upper bound of $x, y$. The element $(x \circ y) \circ y$ is an idempotent with respect to $\vee$ since $[(x \circ y) \circ y] \vee[(x \circ y) \circ y]=([(x \circ y) \circ y] \circ[(x \circ y) \circ y]) \circ[(x \circ y) \circ y]=1 \circ((x \circ y) \circ y)=(x \circ y) \circ y(b y$ (1)). Let $z$ be an idempotent such that $x \preceq z, y \preceq z$. Then $z \vee z=z=(z \circ z) \circ z=1 \circ z$, 
so that $z=1 \circ z=(y \circ z) \circ z=(z \circ y) \circ y$. Further, $[(x \circ y) \circ y] \circ z=[(x \circ y) \circ y] \circ[(z \circ y) \circ y]=$ $(z \circ y)[((x \circ y) \circ y) \circ y]=(z \circ y) \circ(x \circ y)=x \circ((z \circ y) \circ y)=x \circ z=1$, that is, $(x \circ y) \circ y \preceq z$, and $(x \circ y) \circ y$ is the least idempotent above the elements $x$ and $y$. For any element $a \in \operatorname{Sk}[p \vee p, 1]$, the map $a \mapsto a^{p}=a \circ p$ is an involution since

$$
a^{p p}=(a \circ p) \circ p=a \vee p=a \vee p \vee p=a \vee a=a,
$$

and is antitone by (iii). For $a, b \in[p \vee p, 1]$, define $a \wedge b=\left(a^{p} \vee b^{p}\right)^{p}$. Obviously, $([p \vee p, 1], \vee, \wedge)$ is a $q$-lattice. Let us prove that this $q$-lattice is distributive. According to [6], Theorem 2, p.11, a $q$-lattice is distributive iff its skeleton, i.e. the lattice $(\operatorname{Sk}[p \vee p, 1], \vee, \wedge)$, is distributive. Assume on the contrary that $(\operatorname{Sk}[p \vee p, 1], \vee, \wedge)$ is not distributive. Then it contains a sublattice isomorphic to $M_{3}$ or to $N_{5}$.

$\mathrm{C}$ as e 1. Let a lattice from Fig. 1 be a sublattice of $(\operatorname{Sk}[p \vee p, 1], \vee, \wedge)$. Then it is also a sublattice of $\operatorname{Sk}[x, 1]$. Since $a \mapsto a^{x}$ is a dual automorphism on $\operatorname{Sk}[x, 1]$, $(\mathrm{Sk}[x, 1], \vee, \wedge)$ contains a sublattice given in Fig. 2 .

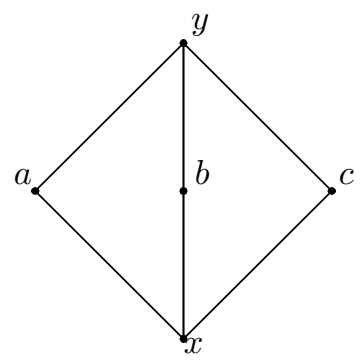

Fig. 1

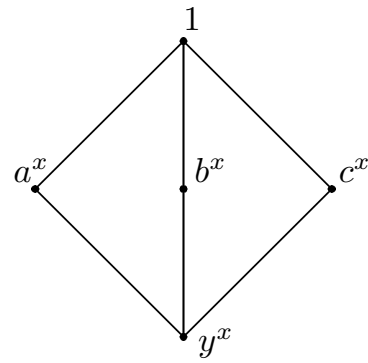

Fig. 2

Then $a^{x} \circ(c \circ x)=a^{x} \circ c^{x}=\left(a^{x} \vee c^{x}\right)^{c^{x}}=1^{c^{x}}=c^{x}, c \circ\left(a^{x} \circ x\right)=c \circ((a \circ$ $x) \circ x)=c \circ(a \vee x)=c \circ a=(c \vee a)^{a}=y^{a}$. Since $\mathcal{A}$ satisfies (4), $c^{x}=y^{a}$ holds. Analogously, interchanging $b$ and $c$ we obtain $b^{x}=y^{a}$, therefore $c^{x}=b^{x}$, hence $c=b$, a contradiction.

$\mathrm{C}$ as e 2. Let us consider a sublattice of $(\operatorname{Sk}[p \vee p, 1], \vee, \wedge)$ from Fig. 3. Then $b^{x} \circ(a \circ x)=b^{x} \circ a^{x}=\left(b^{x} \vee a^{x}\right)^{a^{x}}=1^{a^{x}}=a^{x}, a \circ\left(b^{x} \circ x\right)=a \circ((b \circ x) \circ x)=$ $a \circ(b \vee x)=a \circ b=(a \vee b)^{b}=y^{b}$, hence $a^{x}=y^{b}$. Interchanging $a, c$ we obtain $c^{x}=y^{b}$, therefore $a^{x}=c^{x}$ and $a=c$, a contradiction.

In the sequel we will investigate the variety $\widetilde{\mathcal{W}}=\operatorname{Mod}(\{(1),(2),(3),(4)\})$ of algebras satisfying the identities (1)-(4).

$\mathrm{R}$ e $\mathrm{m}$ ar $\mathrm{k}$ 4.7. In [10], the variety $\mathcal{W}$ of type $(2,0)$ given by the identities $1 \circ x=x$, $x \circ 1=1,(3)$ and (4) was investigated and it was proved that $\mathcal{W}$ is 1 -regular and 3 -permutable. It can be verified that $\widetilde{\mathcal{W}}$ and $N(\mathcal{W})$ coincide. In fact, $1 \circ x=x$ 
is the only non-normal identity among the defining identities of $\mathcal{W}$, and $1 \circ x$ is the assigned term. Hence the normalization $N(\mathcal{W})$ is defined by normal identities $x \circ 1=1,(3),(4)$ together with additional identities $(1 \circ x) \circ y=x \circ y, x \circ(1 \circ y)=x \circ y$, $1 \circ(x \circ y)=x \circ y$. The third identity is our (2), the second identity also holds in $\widetilde{\mathcal{W}}$ since $x \circ(1 \circ y)=1 \circ(x \circ y)=x \circ y$ by (3) and (2), and the first identity can be proved as follows. Let us calculate $((1 \circ x) \circ y) \circ(x \circ y)=x \circ(((1 \circ x) \circ y) \circ y)=$ $x \circ((y \circ(1 \circ x)) \circ(1 \circ x))=(y \circ(1 \circ x)) \circ(x \circ(1 \circ x))=(y \circ(1 \circ x)) \circ 1=1$ and $(x \circ y) \circ((1 \circ x) \circ y)=(1 \circ x) \circ((x \circ y) \circ y)=(1 \circ x) \circ((y \circ x) \circ x)=(y \circ x) \circ((1 \circ x) \circ x)=$ $(y \circ x) \circ((x \circ 1) \circ 1)=(y \circ x) \circ 1=1$. It means $(1 \circ x) \circ y \preceq x \circ y$ and at the same time $(1 \circ x) \circ y \succeq x \circ y$. Since both $(1 \circ x) \circ y$ and $x \circ y$ are skeletal elements they must be equal. So we have verified that $\operatorname{Id}(N(\mathcal{W})) \subset \operatorname{Id}(\widetilde{\mathcal{W}})$. The converse inclusion is also true since in $N(\mathcal{W}), x \circ x=(1 \circ x) \circ x=(x \circ 1) \circ 1=1 \circ 1=1$ holds.

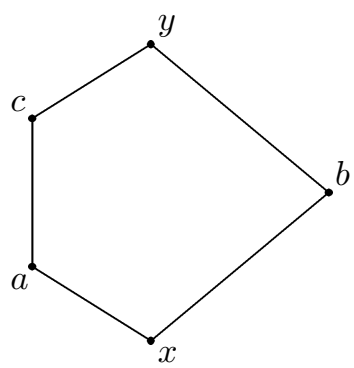

Fig. 3

The following example illustrates that the variety $\widetilde{\mathcal{W}}$ is not 1-regular.

Example 4.8. For $\mathcal{A} \in \widetilde{\mathcal{W}}$, there may exist different congruences $\theta \neq \omega$ (see Fig. 4) such that their congruence kernels coincide, $[1]_{\theta}=[1]_{\omega}$.
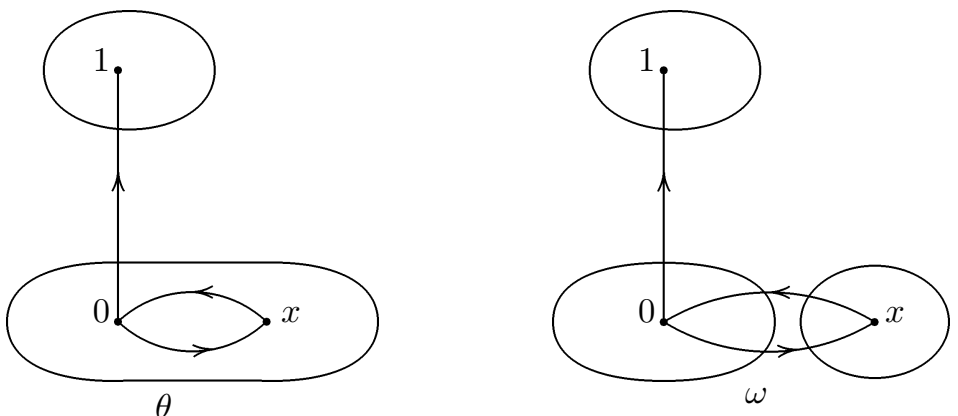

Fig. 4 
Theorem 4.9. Let $\mathcal{A} \in \widetilde{\mathcal{W}}, M \subseteq A$. Then $M$ is a kernel of a congruence $\theta \in \operatorname{Con}(\mathcal{A})$ iff

(K1) $1 \in M$,

(K2) if $x \in M$ and $x \circ y \in M$ then $1 \circ y \in M$.

Pro of. It can be easily seen that for $\theta \in \operatorname{Con}(\mathcal{A}),[1]_{\theta}$ satifies $(\mathrm{K} 1)$ and $(\mathrm{K} 2)$. On the other hand, for $M$ satisfying (K1) and (K2), let us introduce

$$
\theta_{M}=\{\langle x, y\rangle ; x \circ y \in M \text { and } y \circ x \in M\} .
$$

$\theta_{M}$ is obviously reflexive and symmetric. Let $\langle x, y\rangle,\langle y, z\rangle \in \theta_{M}$. Then $y \preceq(x \circ y) \circ y=$ $(y \circ x) \circ x, z \circ y \preceq z \circ((y \circ x) \circ x)=(y \circ x) \circ(z \circ x)$, therefore $(z \circ y) \circ[(y \circ x) \circ(z \circ x)]=1 \in M$. But $z \circ y \in M$, so according to (K2) and (1), $1 \circ[(y \circ x) \circ(z \circ x)]=(y \circ x) \circ(z \circ x) \in M$. Further, $y \circ x \in M$, so again by (K2) and (1), $1 \circ(z \circ x)=z \circ x \in M$. Similarly, $x \circ z \in M$ can be proved, hence $\theta_{M}$ is an equivalence relation.

Now let $\langle x, y\rangle \in \theta_{M}$. Then $x \preceq(x \circ y) \circ y$ gives $z \circ x \preceq z \circ((x \circ y) \circ y)=(x \circ y) \circ(z \circ y)$, which yields $1=(z \circ x) \circ[(x \circ y) \circ(z \circ y)]=(x \circ y) \circ[(z \circ x) \circ(z \circ y)]$ and by $(\mathrm{K} 2), 1 \circ[(z \circ x) \circ(z \circ y)]=(z \circ x) \circ(z \circ y) \in M$. Interchanging $x, y$ we obtain $(z \circ y) \circ(z \circ x) \in M$, therefore $\langle z \circ x, z \circ y\rangle \in \theta_{M}$. Further, $y \circ x \preceq(z \circ x) \circ(y \circ x)=$ $y \circ((z \circ x) \circ x)=y \circ((x \circ z) \circ z)=(x \circ z) \circ(y \circ z),(y \circ x) \circ[(x \circ z) \circ(y \circ z)]=1 \in M$, $y \circ x \in M$. Using (K2) we obtain $1 \circ((x \circ z) \circ(y \circ z))=(x \circ z) \circ(y \circ z) \in M$. Similarly $(y \circ z) \circ(x \circ z) \in M$, therefore $\langle x \circ z, y \circ z\rangle \in \theta_{M}$. Transitivity of $\theta_{M}$ implies the compatibility of $\theta_{M}$. Obviously, $[1]_{\theta_{M}}=\{y ; 1 \circ y \in M\}$. Let us introduce a binary relation $\theta_{M}^{*}=\theta_{M} \backslash\{\langle x, y\rangle,\langle y, x\rangle ; x \in M, y \notin M\}$. Let us verify that $\theta_{M}^{*}$ is a congruence relation on $\mathcal{A}$. Clearly, $\theta_{M}^{*}$ is both reflexive and symmetric. To show transitivity, let $\langle x, y\rangle \in \theta_{M}^{*},\langle y, z\rangle \in \theta_{M}^{*}$, and let $x \in M$. Then $y \in M$ since $\langle x, y\rangle \in \theta_{M}^{*}$, and similarly $z \in M$ since $\langle y, z\rangle \in \theta_{M}^{*}$. Analogously, it follows that $x \in M$ from $z \in M$, therefore $\langle x, z\rangle \in \theta_{M}^{*}$. To prove compatibility of $\theta_{M}^{*}$ it is sufficient (due to transitivity of $\theta_{M}^{*}$ ) to verify the implications $\langle x, y\rangle \in \theta_{M}^{*} \Longrightarrow\langle x \circ z, y \circ z\rangle \in \theta_{M}^{*}$, $\langle x, y\rangle \in \theta_{M}^{*} \Longrightarrow\langle z \circ x, z \circ y\rangle \in \theta_{M}^{*}$. Obviously, $\langle x \circ z, y \circ z\rangle \in \theta_{M}$ and $\langle z \circ y, z \circ x\rangle \in \theta_{M}$ hold. Let $x \circ z \in M$. Then $(x \circ z) \circ(y \circ z) \in M$, and since $M$ satisfies (K2) we obtain $1 \circ(y \circ z)=y \circ z \in M$. Analogously $x \circ z \in M$ follows from $y \circ z \in M$.

Similarly, $z \circ y \in M$ yields $(z \circ y) \circ(z \circ x) \in M$, and again $1 \circ(z \circ x)=z \circ x \in M$. Together, $\theta_{M}^{*}$ is a congruence relation with the congruence kernel $[1]_{\theta_{M}^{*}}=M$.

Definition 4.10. A subset $M \subseteq A, \mathcal{A} \in \widetilde{\mathcal{W}}$ satisfying (K1) and (K2) will be called a deductive system. 
Theorem 4.11. Let $\mathcal{A} \in \widetilde{\mathcal{W}}, a \in A$. Then the deductive system generated by $a$ is

$$
D(a)=\{a\} \cup\{x ; 1 \circ x=x \text { and } a \circ(a \circ \ldots \circ(a \circ x))=1\} .
$$

Proof. Let $M=\{a\} \cup\{x ; 1 \circ x=x$ and $a \circ(a \circ \ldots \circ(a \circ x))=1\}$. If $x \in M$ then either $x=a \in D(a)$, or $1 \circ x=x, a \circ(a \circ \ldots \circ(a \circ x))=1 \in D(a)$. Since $D(a)$ is a deductive system, $a \in D(a)$, we obtain from (K2) $1 \circ x=x \in D(a)$, hence $M \subseteq D(a)$. On the other hand, obviously 1 and $a$ belong to $M$. Let us prove that $M$ is a deductive system. Let $x \in M, x \circ y \in M$. There are two possibilities:

(a) $x=a, x \circ y=a \circ y \in M, a \circ(a \circ \ldots \circ(a \circ y))=1$, therefore $a \circ(a \circ \ldots \circ(a \circ(1 \circ y)))=$ $a \circ(a \circ \ldots \circ(a \circ y))=1$, which yields $1 \circ y \in M$.

(b) $a \circ(a \circ \ldots \circ(a \circ x))=1, a \circ(a \circ \ldots \circ(a \circ(x \circ y)))=1$. Then applying (4) to the second equality gives $x \circ(a \circ(a \circ \ldots \circ(a \circ y)))=1$, i.e. $x \preceq a \circ(a \circ \ldots \circ(a \circ y))$. Multiplying by an element $a$ from the left we obtain $1=a \circ(a \circ \ldots \circ(a \circ x)) \preceq a \circ(a \circ \ldots \circ(a \circ y))$, so $a \circ(a \circ \ldots \circ(a \circ y))=1$ holds. But then $a \circ(a \circ \ldots \circ(a \circ(1 \circ y)))=a \circ(\ldots \circ(a \circ y))=1$, hence $1 \circ y \in M$. Obviously, $M$ is the least deductive system containing $M$.

Rem ark 4.12. Let $\mathcal{A} \in \widetilde{\mathcal{W}}$. An element $a \in A$ is skeletal iff $1 \circ a=a$. The set of all skeletal elements forms the skeleton $\operatorname{Sk} \mathcal{A} \in \mathcal{W}$. ( $\operatorname{Sk} \mathcal{A}, \circ, 1)$ is a subalgebra in $\mathcal{A}$, and any interval $[x, 1]$ in $\operatorname{Sk} \mathcal{A}$ is a lattice with an antitone involution.

Theorem 4.13. Let $\mathcal{A}=(A, \circ, 1)$ be a finite algebra in $\widetilde{\mathcal{W}}$. Then $\mathcal{A}$ is subdirectly irreducible if and only if $(\mathcal{A}, \preceq)$ is a chain and $|A \backslash \operatorname{Sk} \mathcal{A}| \leqslant 1$.

Proof. If $A=\operatorname{Sk} \mathcal{A}$ then the proof follows by [10]. If $A \backslash \operatorname{Sk} \mathcal{A}$ is an at least two-element set with $x, y \in A \backslash \operatorname{Sk} \mathcal{A}$ then $\theta_{1}=\omega \cup\{\langle 1 \circ x, x\rangle,\langle x, 1 \circ x\rangle\}$, $\theta_{2}=\omega \cup\{\langle 1 \circ y, y\rangle,\langle y, 1 \circ y\rangle\}$ are congruence relations, $\theta_{1}, \theta_{2} \neq \omega, \theta_{1} \cap \theta_{2}=\omega$, hence $(A, \circ, 1)$ is subdirectly reducible.

Theorem 4.14. Let $\mathcal{A}=(A, \oplus, \neg, 0) \in N(\underline{M V})$. Define $x \circ y:=\neg x \oplus y$ and $1=\neg 0$. Then $\mathcal{L}(\mathcal{A})=(A, \vee, \wedge, \circ, 1,0)$ is a bounded distributive $q$-lattice with sectionally antitone involutions satisfying the identity (4).

Pr o of. Let us prove that the mapping ${ }^{p}: \operatorname{Sk}[p \vee p, 1] \rightarrow \operatorname{Sk}[p \vee p, 1]$ where $x \mapsto$ $x^{p}=\neg x \oplus p, p \in A$, is an antitone involution. Indeed, we have $x^{p p}=\neg(\neg x \oplus p) \oplus p=$ $x \vee p=x \vee x=x$. Further, if $x \preceq y$ then $\neg x \succeq \neg y$ (since $x, y$ are skeletal), hence $x^{p}=\neg x \oplus p \succeq \neg y \oplus p=y^{p}$ for all $x, y \in \operatorname{Sk}[p \vee p, 1]$. If $x, y \in[p, 1]$ and $y \vee y=x \vee x=x$ then $\neg x \oplus p=\neg(y \vee y) \oplus p=\neg y \oplus p$ since $N(\underline{M V})$ satisfies all normal identities of $\underline{M V}$. By the same argument we have $x \circ(y \circ z)=\neg x \oplus(\neg y \oplus z)=\neg y \oplus(\neg x \oplus z)=y \circ(x \circ z)$. 
Theorem 4.15. Let $\mathcal{L}=(L, \vee, \wedge, \circ, 1,0)$ be a bounded q-lattice with sectionally antitone involutions that satisfies the identity (4). Define $\neg x:=x \circ 0, x \oplus y:=$ $(x \circ 0) \circ y$. Then $\mathcal{A}(\mathcal{L})=(L, \oplus, \neg, 0) \in N(\underline{M V})$.

Proof. We shall verify the axioms (N1)-(N3), (N5), (N6), (N4'), (N9) and (N10). First, let us prove that $x \circ y=x \circ((y \circ 0) \circ 0)$ for all $x, y \in L$. Indeed, $(y \circ 0) \circ 0=(y \vee 0)^{0} \circ 0=\left((y \vee 0)^{0} \vee 0\right)^{0}=(y \vee 0)^{00}=y \vee 0$, hence $x \circ((y \circ 0) \circ 0)=$ $x \circ(y \vee 0)=(x \vee y \vee 0)^{y \vee 0}=(x \vee y)^{y \vee y}=x \circ y$. Using this identity we compute

$(\mathrm{N} 1):(x \oplus y) \oplus z=(((x \circ 0) \circ y) \circ 0) \circ z=(((x \circ 0) \circ y) \circ 0) \circ((z \circ 0) \circ 0)=$ $(z \circ 0) \circ((((x \circ 0) \circ y) \circ 0) \circ 0)=(z \circ 0) \circ((x \circ 0) \circ y)=(x \circ 0) \circ((z \circ 0) \circ((y \circ 0) \circ 0))=$ $(x \circ 0) \circ((y \circ 0) \circ((z \circ 0) \circ 0)=(x \circ 0) \circ((y \circ 0) \circ z)=x \oplus(y \oplus z)$,

$(\mathrm{N} 2): x \oplus y=(x \circ 0) \circ y=(x \circ 0) \circ((y \circ 0) \circ 0)=(y \circ 0) \circ((x \circ 0) \circ 0)=(y \circ 0) \circ x=y \oplus x$,

$(\mathrm{N} 3): 0 \oplus 0=(0 \circ 0) \circ 0=(0 \vee 0)^{0} \circ 0=0^{0} \circ 0=1 \circ 0=(1 \vee 0)^{0}=1^{0}=0$,

$(\mathrm{N} 5): x \oplus \neg 0=(x \circ 0) \circ(0 \circ 0)=(x \circ 0) \circ 1=1=0 \circ 0=\neg 0$,

$(\mathrm{N} 6): \neg(\neg x \oplus y) \oplus y=(x \circ y) \circ y=(y \circ x) \circ x=\neg(\neg y \oplus x) \oplus x$,

$\left(\mathrm{N} 4^{\prime}\right): \neg \neg 0=(0 \circ 0) \circ 0=1 \circ 0=0$,

$(\mathrm{N} 9): x \oplus y \oplus 0=((x \oplus y) \circ 0) \circ 0=(((x \circ 0) \circ y) \circ 0) \circ 0=(x \circ 0) \circ y=x \oplus y$,

(N10): $\neg \neg \neg x=((x \circ 0) \circ 0) \circ 0=x \circ 0=\neg x$.

Corollary 4.16. Let $\mathcal{A}=(A, \circ, 1)$ be an algebra satisfying (1)-(4). Let $p \in A$ with $1 \circ p=p$ and define $\neg_{p} x:=x \circ p, x \oplus_{p} y:=(x \circ p) \circ y$. Then the algebra $\left([p, 1], \oplus_{p}, \neg p, p\right)$ belongs to $N(\underline{M V})$.

\section{References}

[1] Anderson, M., Feil, T.: Lattice-ordered groups. An Introduction. D. Reidel., Dordrecht, 1988.

[2] Chang, C. C.: Algebraic analysis of many valued logics. Trans. Amer. Math. Soc. 88 (1958), 467-490.

[3] Chang, C. C.: A new proof of the Eukasziewicz axioms. Trans. Amer. Math. Soc. 93 (1959), 74-80.

[4] Cignoli, R.: Free lattice-ordered abelian groups and varieties of $M V$-algebras. Proc. IX. Latin. Amer. Symp. Math. Logic, Part 1, Not. Log. Mat. 38 (1993), 113-118.

[5] Cignoli, R.L.O., D'Ottaviano, I. M.L., Mundici, D.: Algebraic Foundations of ManyValued Reasoning. Kluwer, Dordrecht, 2000.

[6] Chajda, I.: Lattices in quasiordered sets. Acta Univ. Palacki. Olomuc., Fac. Rerum Nat., Math. 31 (1992), 6-12.

[7] Chajda, I.: Congruence properties of algebras in nilpotent shifts of varieties. General Algebra and Discrete Mathematics (K. Denecke, O. Lüders, eds.), Heldermann, Berlin, 1995, pp. 35-46.

[8] Chajda, I.: Normally presented varieties. Algebra Universalis 34 (1995), 327-335.

[9] Chajda, I., Graczyńska, E.: Algebras presented by normal identities. Acta Univ. Palacki. Olomuc., Fac. Rerum Nat., Math. 38 (1999), 49-58. 
[10] Chajda, I., Halaš, R., Kühr, J.: Distributive lattices with sectionally antitone involutions. To appear in Acta Sci. Math. (Szeged).

[11] Mel'nik, I. I.: Nilpotent shifts of varieties. Math. Notes 14 (1973), 692-696. (In Russian.)

[12] Mundici, D.: Interpretation of $\mathrm{AF} C^{*}$-algebras in Lukasiewicz sentential calculus. J. Funct. Anal. 65 (1986), 15-63.

[13] Mundici, D.: $M V$-algebras are categorically equivalent to bouded commutative $B C K$ algebras. Math. Japon. 31 (1986), 889-894.

[14] Rachuinek, J.: $M V$-algebras are categorically equivalent to a class of $D R l_{1(i)^{-}}$-semigroups. Math. Bohem. 123 (1998), 437-441.

Author's address: I. Chajda, R. Halaš, J. Kühr, A. Vanžurová, Dept. of Algebra and Geometry, Palacký University Olomouc, Tomkova 40, 77900 Olomouc, Czech Republic. 